\title{
Solvent-Engineered Stress in Nanoscale Materials
}

Shaun Mills ${ }^{\dagger,}$, Chiara Rotella ${ }^{\dagger, \perp}$, Eoin K. McCarthy ${ }^{\perp}$, David J. Hill ${ }^{\phi}$, Jing-Jing Wang ${ }^{\perp}$, John F. Donegan ${ }^{\S, \perp}$, James F. Cahoon ${ }^{\phi}$, John E. Sader ${ }^{\perp, \$}$, \& John J. Boland ${ }^{*,+, \perp}$

†School of Chemistry, Trinity College Dublin, Dublin 2, Ireland

${ }^{\S}$ School of Physics, Trinity College Dublin, Dublin 2, Ireland

${ }^{\perp}$ AMBER Research Centre and the Centre for Research on Adaptive Nanostructures and Nanodevices (CRANN), Trinity College Dublin, Dublin 2, Ireland

${ }^{\phi}$ Department of Chemistry, University of North Carolina at Chapel Hill, Chapel Hill, North Carolina 27599-3290, United States

ARC Centre of Excellence in Exciton Science, School of Mathematics and Statistics, University of Melbourne, Victoria, 3010, Australia

Keywords: Nanomaterials, surface passivation, functionalisation, stress, strain-engineering, ink-jet

ABSTRACT: Nanoscale materials are frequently coated with surface stabilisation layers during growth that prevent flocculation in solution and facilitate processing technologies such ink-jet device printing. Here, we show that few-nanometer thick stabilisation layers typically used swell in the presence of certain solvents and impart significant stresses into the nanomaterial that remains even after the solvent has evaporated. Solvent-swelling of the surface layer dramatically enhances nanomaterial-substrate adhesion via the collapse of the stabilisation layer during solvent evaporation, preventing stress relaxation. We demonstrate stress modulation of $\mathrm{Ag}, \mathrm{Au}$ and $\mathrm{Si}$ nanowires functionalised with surface polymers and surfactant layers and detect strain levels of between $0.1 \%$ and $0.6 \%$ using AFM mechanical measurement and Raman spectroscopy. Dry-transferred nanowires exhibit poor adhesion and show no evidence of incorporated stress but become stressed immediately following solvent 
exposure. Strain engineering is demonstrated by coating nanowires with few nanometer thick solvent-responsive polymer layers.

\section{INTRODUCTION}

Nanoscale materials, by their very nature, are characterised by extremely large surface-tovolume ratios so that surface energy makes a significant contribution to their overall energy. For this reason, nanomaterials are prone to agglomeration under solvent processing conditions, potentially resulting in the loss of unique nanoscale properties ${ }^{1}$. This is particularly important for ink-jet printing of devices such as batteries. Steps taken to prevent agglomeration in the solution-based synthesis and processing include the incorporation of surface polymer or functionalisation layers that represent steric and/or electrostatic barriers to agglomeration ${ }^{2,3}$. Whilst it is well recognised that these surface agents affect the size and shapes of grown nanomaterials ${ }^{4}$, and hence size-dependent properties, little consideration has been given to the possible interaction of these surface layers with the solvent medium and their influence on nanomaterial properties.

Here in this work we show that polymer and long-chain functionalisation layers frequently employed to stabilise nanowire systems swell in the presence of certain solvents to impart stress into the nanomaterial that is easily detected by mechanical measurement and Raman spectroscopy. Dry-processed nanowires or wires exposed to non-swelling solvents showed no evidence of incorporated stress. The presence of stress can also be inferred from the adhesion properties of nanowires following drop-casting from solution. Strong adhesion to the substrate prevents stress relaxation in the solvent-deposited wires. Since it is well known that electrical ${ }^{5}$, optical $^{6,7}$ and thermal properties ${ }^{8,9}$ are affected by stress, solvent processing is a potential route to engineer nanoscale material properties. 


\section{EXPERIMENTAL SECTION}

Sample Preparation. AgNWs were purchased from Seashell Technology (http://www.seashelltech.com/). AuNWs were purchased from Sigma Aldrich (http://www.sigmaaldrich.com/catalog/product/aldrich/716952?lang=en\&region=IE) . SiNWs were grown at $650{ }^{\circ} \mathrm{C}$ in a home-built, hot wall chemical vapor deposition system $^{24}$. Au catalysts of diameters $50-100 \mathrm{~nm}$ (BBI international) were immobilized on $\mathrm{Si} / \mathrm{SiO}_{2}$ wafers (NOVA Electronics) with a polylysine solution (Sigma-Aldrich) and subsequently cleaned in a UV-Ozone cleaner before insertion into the CVD. Reactions were carried out at a total pressure of 20 Torr, with $0.5 \mathrm{sccm} \mathrm{SiH}_{4}$ (Voltaix), $3.2 \mathrm{sccm} \mathrm{HCl}$ (Matheson TriGas, 5N), and $200 \mathrm{sccm} \mathrm{H}_{2}$ (Matheson TriGas, $5 \mathrm{~N}$ semiconductor grade). SiNWs were coated using aqueous solutions of PVP (Sigma-Aldrich) at various concentrations to get the required thickness (35nm). The PVP was spun cast onto the nanowires at 4000 RPM (Laurell Technologies) and dried at $100{ }^{\circ} \mathrm{C}$ on a hot plate for one minute.

To make mechanical measurements on nanowires (NWs), trenches were defined in $\mathrm{Si} / \mathrm{SiO}_{2}$ substrates. Trenches were defined using electron beam lithography (EBL) and fabricated using reactive ion etching. Trenches are $2 \mu \mathrm{m}$ wide and $250 \mathrm{~nm}$ deep giving an optimum trench width to wire diameter of around 30 times $^{12}$. NWs were deposited using solvent- and dry-deposition techniques as discussed in the main text. The clamping length of the NWs was defined using platinum electron-beam-induced deposition (Pt-EBID).

Mechanical Measurement. $75 \mathrm{kHz}$ budget sensor tips with a nominal spring constant of 3 $\mathrm{N} / \mathrm{m}$ were used. The calibration procedure for these tips can be found elsewhere ${ }^{11}$. An Asylum MFP-3D atomic force microscope with a lateral lithography suite and closed x-y loop was used to elastically load the nanowires in ambient conditions at room temperature. The set-point force used was typically $500 \mathrm{mV}$. Prior to the measurement the long axis of the cantilever was aligned 
to be parallel with the long axis of the NW. This was to prevent slipping of the AFM tip on the NW during the mechanical manipulation.

For NWs dispersed on blank $\mathrm{SiO}_{2}$ substrates, the mechanical procedure was similar to that above. The difference being that the AFM tip was positioned to sit on or just above the $\mathrm{SiO}_{2}$ surface as opposed to below the x-y plane of the NW as in the 3-point bending experiment.

Raman Measurement. The Raman analysis was performed under a back-scattering geometry on a Renishaw Invia micro-Raman spectrometer equipped with a Charged Coupled Device (CCD) detector. A 3000/mm grating was used to provide high spectral resolution $\left(<0.2 \mathrm{~cm}^{-1}\right)$. A $488 \mathrm{~nm}$ laser was employed as the excitation source. The beam was focussed to a spot about $500 \mathrm{~nm}$ diameter by means of an M Plan Apo objective with 100x magnification and 0.7 numerical aperture, and then incident on the sample. The measured power at the sample was approximately $3.2 \mathrm{~mW}$. The Raman spectrometer was calibrated before each experiment using the sharp Raman peak of bulk silicon at $520 \mathrm{~cm}^{-1}$.

The polarization of excitation laser was set as shown in Figure 6. The orientation of the SiNWs are random and not parallel to the polarization direction. The Raman spectra were analysed by peak fitting procedure by using PstVoigtl function under Spectroscopy category in the software OriginPro 2017.

\section{RESULTS AND DISCUSSION}

Initial stress state of Ag and Si nanowires. To illustrate the behaviour of polymer-stabilized nanowires, the mechanical properties of Ag nanowires (NWs) deposited using solvent-based and dry-transfer techniques are studied. In the former, nanowires pre-dispersed in isopropanol (IPA) are drop-cast $(5 \mu \mathrm{Ls})$ or ink-jet deposited (Microdrop Gantry, 300pL) onto a $\mathrm{SiO}_{2}$ substrate pre-patterned with trenches to facilitate mechanical measurement. Following solvent evaporation, nanowires are found deposited on the substrate, some of which span trenches as 
depicted in Figure 1a. The dry-transfer technique involves the use of a PDMS stamp. Wires pre-dispersed in IPA are drop cast onto PDMS and after solvent evaporation the wires are mechanically transferred onto a trench-patterned substrate (Figure 1b). In each case optical microscopy is used to select NWs suited for mechanical measurement after which mechanical clamps are deposited by Pt-EBID as shown in Figure 2a.

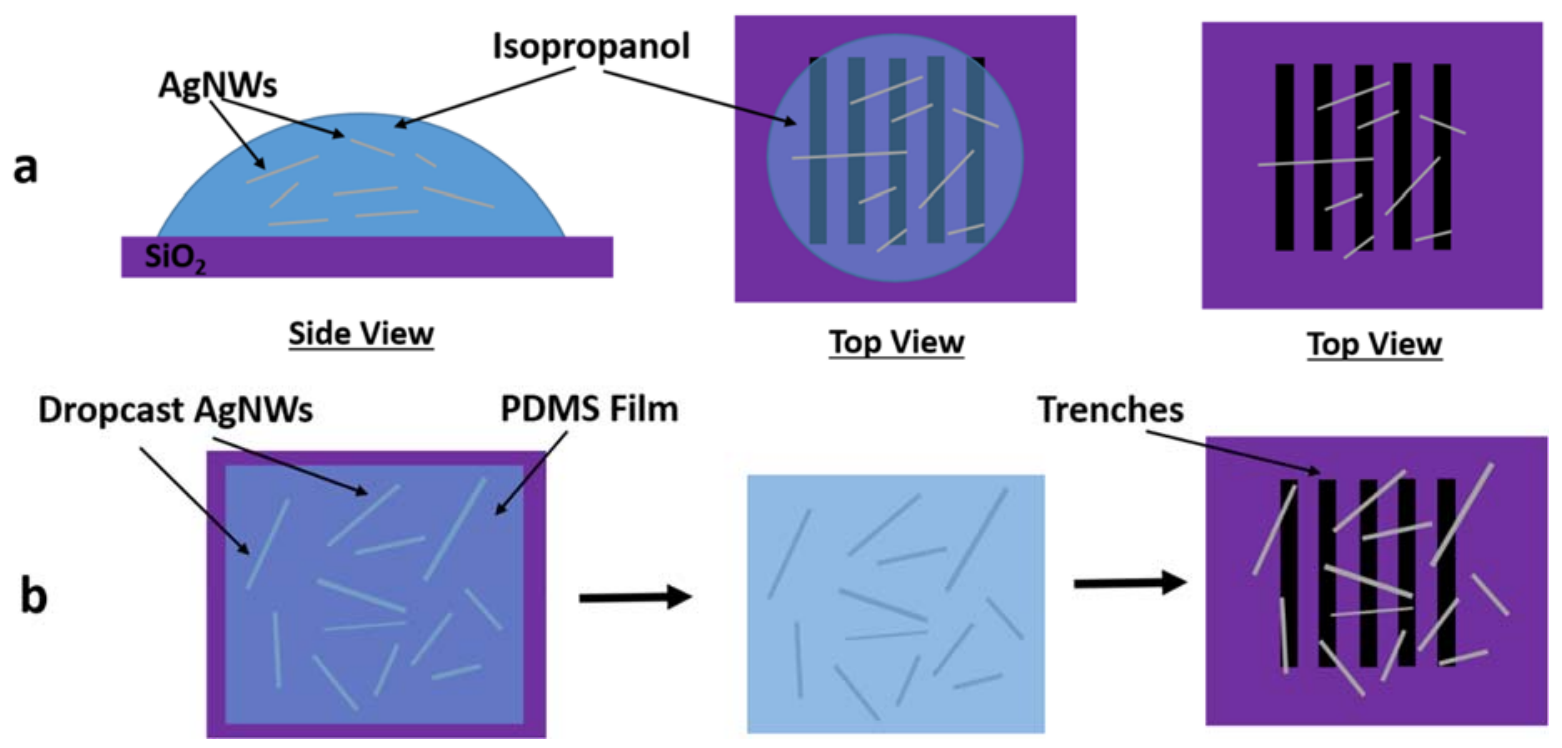

Figure 1. Nanowire deposition techniques. a, Schematic of solvent-deposition of AgNWs. b, Schematic of dry-deposition of AgNWs.

Figure $2 \mathrm{~b}$ shows typical force-displacement $(F-d)$ loading/unloading curves for a solventdeposited AgNW loaded elastically at its mid-point. Prior to loading the tip experiences no force. Upon wire contact and loading an increasing force response is observed. After a userdefined displacement of $110 \mathrm{~nm}$, the tip reverses direction and unloads the wire, resulting in a 

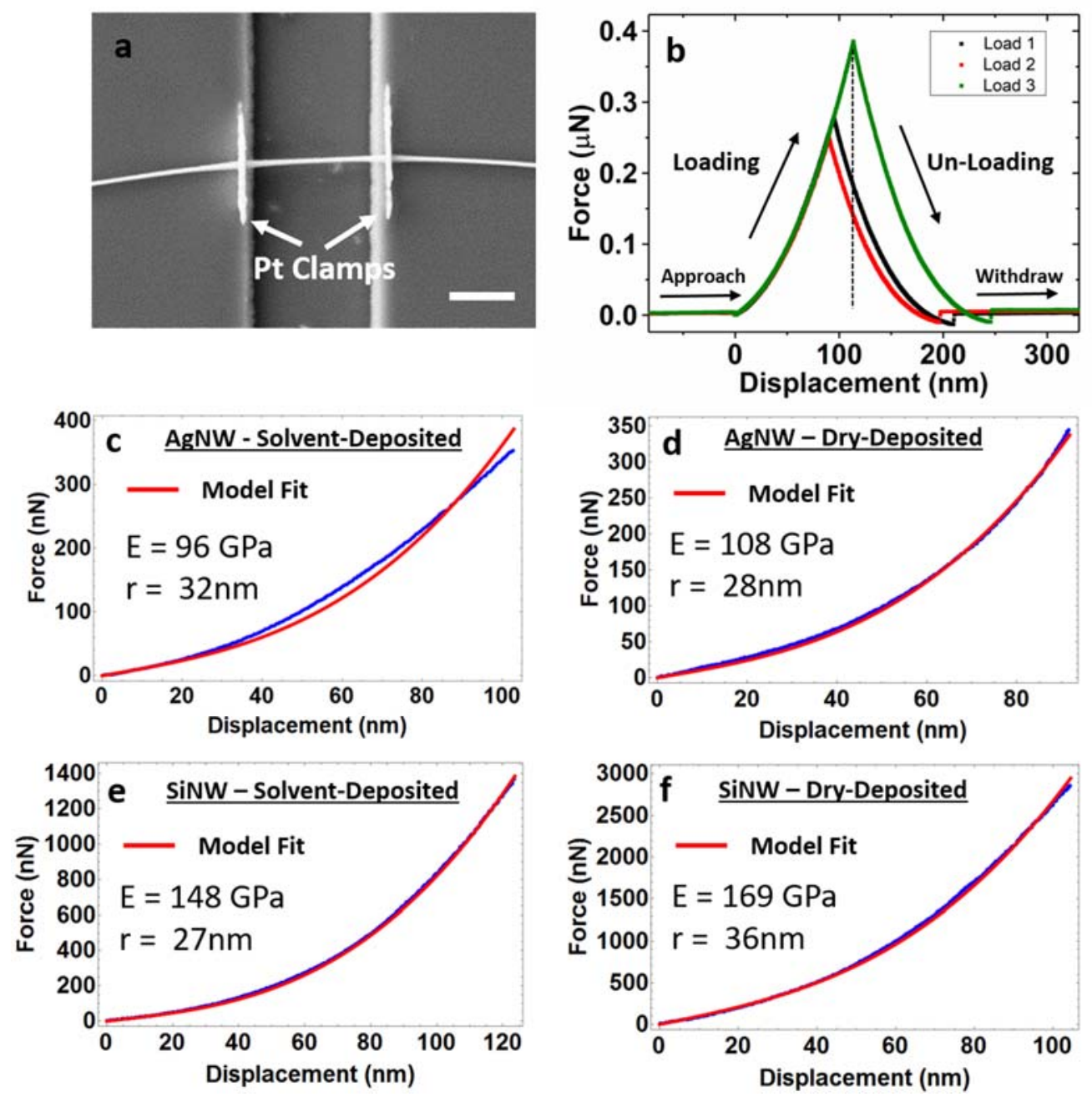

Figure 2. F-d response of $\mathrm{Ag}$ and SiNWs when deposited with and without solvent interactions. a, SEM image of a AgNW suspended over a trench with Pt clamps, scale bar 1 $\mu \mathrm{m}$. b, Repeatable F- $d$ curves for the loading of the AgNW in a. c, F- $d$ response for a solventdeposited AgNW fitted to the standard model (red curve). d, F- $d$ response for a dry-deposited AgNW fitted to the standard model. e, $F-d$ curve for a solvent-deposited SiNW fitted to the standard model. f, $F-d$ curve for a dry-deposited SiNW fitted to the standard model.

decreasing force response. The slight force dip when the tip separates from the NW is due to wire-tip adhesion after which the force returns to its preload value. All manipulations are elastic, inelastic damage would appear as an abrupt change in the slope of the loading curve. The loading curve of the $F-d$ data is generally fitted to the standard model first introduced by Heidelberg et $a l^{10}$. This standard model allows the complete description of the elastic properties of doubly-clamped beams ${ }^{11,12}$ comprised of an initial linear response that accounts for elastic 
beam bending and a cubic term that accounts for stretching of the beam at larger displacements ${ }^{10}$. Figure $2 \mathrm{c}$ shows that the $F$ - $d$ response from solvent-deposited AgNWs is not well described by the standard model. Previously we conducted a very detailed analysis of the issues related to the inability of the strained wires to be described by the standard model ${ }^{13}$. The initial part of the loading curve remains linear up to larger displacements than predicted by the model, indicating the presence of a tensile stress ${ }^{13}$ within the solvent-deposited wire and contrasts with the behaviour found for dry-deposited AgNWs (Figure 2d) where the F-d response is accurately described by the standard model. A similar study involving SiNWs revealed that the mechanical response of solvent- and dry-deposited NWs are both well described by the standard model (Figure $2 \mathrm{e}, \mathrm{f}$ ) indicating that neither wire incorporates stress.

To explain the different behaviours of $\mathrm{Ag}$ and SiNWs in the presence of solvent it is necessary to consider the different surface stabilisation layers. The AgNWs are synthesised using a polyol process $^{14}$ in which the polymer polyvinylpyrollidone (PVP) is used to promote growth of the nanowire in 1-dimension and acts as a stabilising agent to prevent agglomeration, whereas SiNWs contain an outer layer of oxide (see TEM images in Figure S1a and b). The different behaviours is likely attributable to the ability of the PVP layer on the AgNWs to swell in presence of solvent, whereas the oxide layer on SiNWs is impervious to solvent. This hypothesis is corroborated by the fact that AgNWs processed in methanol, ethanol and water, solvents known to swell PVP, result in the incorporation of stress; whereas when AgNWs are exposed to solvents not known to swell PVP, such as heptane and hexane, there is no incorporation of stress (see Figure S2). If solvent induced swelling of the surface layer is the origin of this stress in AgNWs, how then is the stress state retained after the solvent has evaporated?

Adhesion of AgNWs and SiNWs to $\mathrm{SiO}_{2}$. To answer this question, we examined the adhesion properties of the two wires systems, by depositing them on blank $\mathrm{SiO}_{2}$ substrates using both 
deposition methods. Figure 3a shows that AgNWs are strongly adhered to the surface whenever they are solvent-deposited. When loaded with an AFM tip, the main body of the NW remains rigidly adhered to the substrate and the tip fractures the NW at the point of loading (see inset in Figure 3b). In contrast, both Ag and Si dry-deposited NWs are weakly adhered to the surface and slide during the manipulation process. Crucially, we find that solvent-deposited SiNWs are also weakly adhered and slide on the surface (Figure $3 \mathrm{f}$ ). Weakly adhered NWs have the capacity to relax on the surface so that the wires are in a near zero-stress state prior to clamping and mechanical measurement. Figure $3 i$ shows the $F-d$ response observed during the fracture of a $\mathrm{AgNW}$; after the fracture of the NW the force returns to its preload value. In contrast, Figure $3 \mathrm{j}$ shows the F- $d$ response observed for a sliding AgNW, where the force does not return to its preload value as the tip slides the NW along the surface. Evidently, in the case of solvent processed AgNWs the swollen PVP layer becomes rigidly adhered to the substrate and the wires remain under stress after solvent evaporation. Repeat mechanical measurements show that the wires remain adhered indefinitely and heating to $150^{\circ} \mathrm{C}$ for 5 minutes did not affect wire adhesion. Enhanced adhesion is due to the swollen and softened PVP layer which increases the wire's ability to conform to the nanoscale roughness of the substrate (see below). This is corroborated by the fact that the adhesion of AgNWs dispersed in swelling solvents such as water and ethanol is greater than AgNWs dispersed in non-swelling solvents such as heptane and hexane (see Figure S3). 


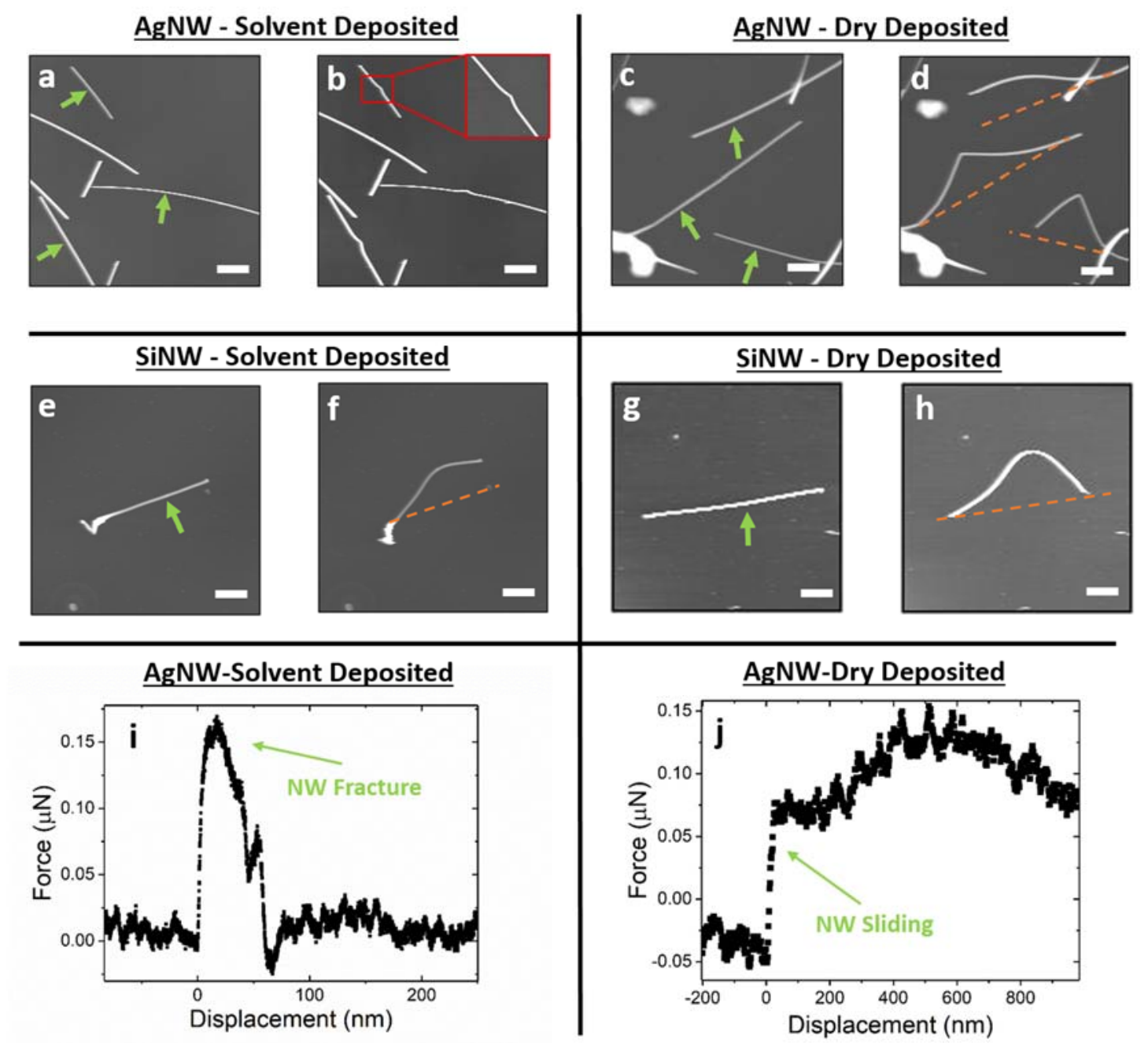

Figure 3. Adhesion of $\mathrm{Ag}$ and $\mathrm{SiNWs}$ to $\mathrm{SiO}_{2}$. AFM images of $\mathrm{AgNWs}$ and $\mathrm{SiNWs}$ on $\mathrm{SiO}_{2}$ before and after being manipulated with an AFM tip. NWs were deposited using different deposition methods. Images a,c,e,g, are wires before manipulation with the green arrows defining the loading path. a,c, AgNWs deposited by solvent and dry methods respectively, scale bars $1 \mu \mathrm{m}$. b, d, The corresponding wires after manipulation with the inset in (b) showing a reduced scan image of a fractured AgNW, scale bars $1 \mu \mathrm{m}$. e,g, SiNWs deposited by solvent and dry methods respectively with $\mathbf{f}$ and $\mathbf{h}$ showing the same wires after having being manipulated with the AFM tip, scale bars $500 \mathrm{~nm}$. i, $F-d$ response observed when manipulating a solvent-deposited AgNW. j, F-d response observed when manipulating a dry-deposited AgNW.

Comparative experiments with AuNWs. To explore the generality of this phenomenon, we analysed the adhesion of gold nanowires coated with cethyltrimethylammoniumbromide (CTAB), a surfactant which serves the same purpose as the PVP for AgNWs ${ }^{15}$. We found that similar to the AgNW case, solvent-deposited AuNWs are strongly adhered to the substrate and that mechanical measurements reveal the presence of tensile stress. When dry-deposited on a 
$\mathrm{SiO}_{2}$ surface the AuNWs are mobile during manipulation with an AFM tip. The results in Supplemental Figure S4 show that the CTAB coating on the AuNWs interacts with solvent $\left(\mathrm{H}_{2} \mathrm{O}\right.$ in this case $)$ in a manner very similar to that found for PVP-coated AgNWs. It is well established that $\mathrm{CTAB}\left[\mathrm{CH}_{3}\left(\mathrm{CH}_{2}\right)_{15} \mathrm{~N}^{+}\left(\mathrm{CH}_{3}\right)_{3}\right] \mathrm{Br}^{-}$is a cationic surfactant that shapes the growth of Au wires by forming a surface head-to-tail bilayer in which the positively charged trimethylammonium head groups positioned at the wire-CTAB interface and at the outer exposed surface of the functionalised wire ${ }^{16}$. It is also well established that these headgroups become hydrated in water by interacting with the dipoles of the solvent leading to a volume expansion, which we suggest imparts stress to the layer and wire.

Solvent induced polymer swelling. The interplay between adhesion and solvent swelling of the PVP layer is further studied by considering the behaviour of individual dry-deposited AgNWs before and after exposure to solvent. Figure $4 \mathrm{a}$ and $\mathrm{b}$ show that the dry-deposited $\mathrm{AgNW}$ is easily manipulated on the surface and hence capable of relaxing into a minimally stressed state. The manipulation, which creates a clearly visible deformation of the wire, is completely eliminated in Figure $4 \mathrm{c}$ after a drop of isopropanol is placed over the wire and allowed to evaporate. In the presence of solvent there is minimal adhesion between the wire and the substrate so that manipulation in the solvent environment causes the wires to become dislodged from the substrate. In the case of Figure $4 b, c$ the reduced adhesion allows the intrinsic stiffness of the wire to straighten out the bend created by the earlier dry manipulation. The mechanical response of the wires is dramatically different after the solvent evaporates. Subsequent attempts to manipulate the wire fail, the wire is now rigidly adhered to the surface. AFM height measurements along the length of the wire before and after solvent exposure reveal that the wire diameter decreased from $61.96 \pm 4.04 \mathrm{~nm}$ to $59.62 \pm 3.95 \mathrm{~nm}$ (see Figure 4e). This is consistent with the collapse of the swollen PVP layer under the compressive forces of 
the attractive interaction between the wire and the substrate, resulting in an increased contact area and enhanced adhesion. Mechanical testing of the wire after solvent exposure reveals the wire is rigidly clamped to the substrate and $F-d$ measurement indicates the presence of stress (Figure 4f). Repeated manipulations ultimately led to the fracture of the wire at one of the pinning clamps, a typical failure mechanism for a double-clamped beam (Figure 4d). The data in Figure 4 conclusively points to the role of the solvent in incorporating stress into the wire and fixing this stress through enhanced adhesion of the PVP layer to the substrate. The $\mathrm{SiO}_{2}$ substrates used in this study have an rms roughness of $<0.5 \mathrm{~nm}$, which is typical of this commonly used substrate. Our expectation is that our adhesion- driven phenomenon becomes less pronounced as the surface roughness increases. The spatial length scale is also important; a low frequency roughness even of pronounced amplitudes will not impact adhesion provided the wires are mechanically compliant on this lengthscale. 

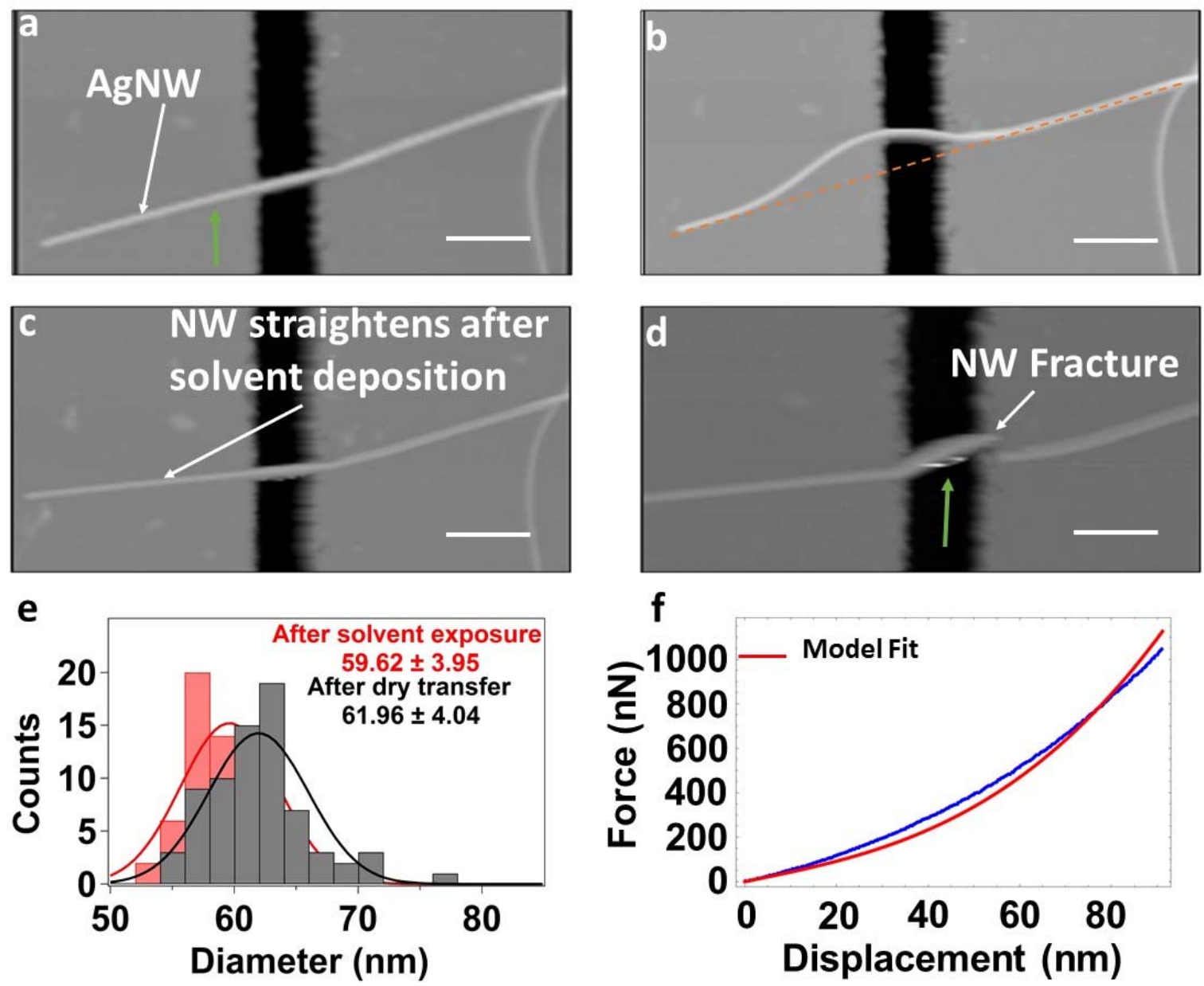

Figure 4. IPA deposited on dry-deposited AgNW. a, AFM image of a dry-deposited AgNW showing manipulation path (green arrow). $\mathbf{b}$, Movement of AgNW indicating wire is weakly adhered to the surface. c, Subsequent straightening out of the NW upon exposing to solvent drop. d, Fractured NW after subsequent manipulation showing pinned NW, scale bars $2 \mu \mathrm{m}$. e, Change in the diameter distribution along length of the wire before (a) and after (c) exposure to IPA solvent. The histograms involve a topographic analysis of 70 sequential cross-sections each perpendicular to the wire, with the analysis extending along the total length of the wire. The set-point force is $550 \mathrm{mV}$ in each case. The mean and width values are derived from Gaussian fits to these data. f, $F-d$ curve fit to the standard model after IPA exposure.

We now estimate the level of strain that can be induced in the AgNWs by a few nanometrethick PVP surface layer. It is well known that polymers swell in solvent media ${ }^{17,18}$. The extent to which a polymer swells depends heavily on the cross-linking of the polymer. Assuming isotropic swelling of the polymer we estimate the strain in the NW as follows:

$$
\varepsilon_{\text {wire }}=-2\left(\frac{h_{\text {film }}}{R_{\text {wire }}}\right)\left(\frac{E_{\text {film }}}{E_{\text {wire }}}\right) \varepsilon_{f i l m}
$$


where, $\varepsilon_{\text {wire }}$ and $\varepsilon_{\text {film }}$ are the strain of the nanowire and polymer film respectively, $h_{\text {film }}$ is the thickness of the polymer layer, $R_{\text {wire }}$ is the wire radius and $E$ is the Young's modulus of the wire and polymer coating. This formula provides the basis for engineering strain in nanowire materials. In the present case, the PVP film has an average thickness of $3 \mathrm{~nm}$ (Figure S1a). The Young's modulus of the PVP surface layer is unknown and difficult to measure. For the polyol process the most common molecular weight used is $55,000^{14,19}$. PVP with a molecular weight of 58,000 is reported to have a Young's modulus of $2.5 \mathrm{GPa}^{20}$. Whereas expansions of $>1000 \%$ in solvents have been reported ${ }^{21}$, the degree of swelling is unknown.

We estimate the level of strain required to explain the $F-d$ response in Figure 5 by generating a synthetic $F-d$ curve using the model outlined by Yaish et $a l^{22}$. This model is an extension of the standard mode ${ }^{10}$ to include the effects of stress. Figure $5 b$, shows that the experimental $F$ $d$ response from the solvent-deposited wire in Figure 5a is poorly described by the standard model, however, it is well described by the synthetic F-d curve with a strain level of $0.1 \%$. Also shown is the $F-d$ response that would be obtained for the same NW had it not been affected by an initial stress. Given a wire strain of $0.1 \%$, using Eq. (1) and assuming a PVP layer with Young's modulus of $2 \mathrm{GPa}$ and thickness $3 \mathrm{~nm}$, a AgNW radius of $32 \mathrm{~nm}$ (Figure 2c) and modulus of $75 \mathrm{GPa}$, we estimate $\varepsilon_{\text {film }} \approx 0.2$ corresponding to a $20 \%$ swelling of the PVP layer. This analysis demonstrates that a modest swelling of the outer polymer/surfactant coatings creates significant stress levels and that a careful choice of solvent and passivation layer is a route to strain engineering. We illustrate this now for the case of SiNWs. 

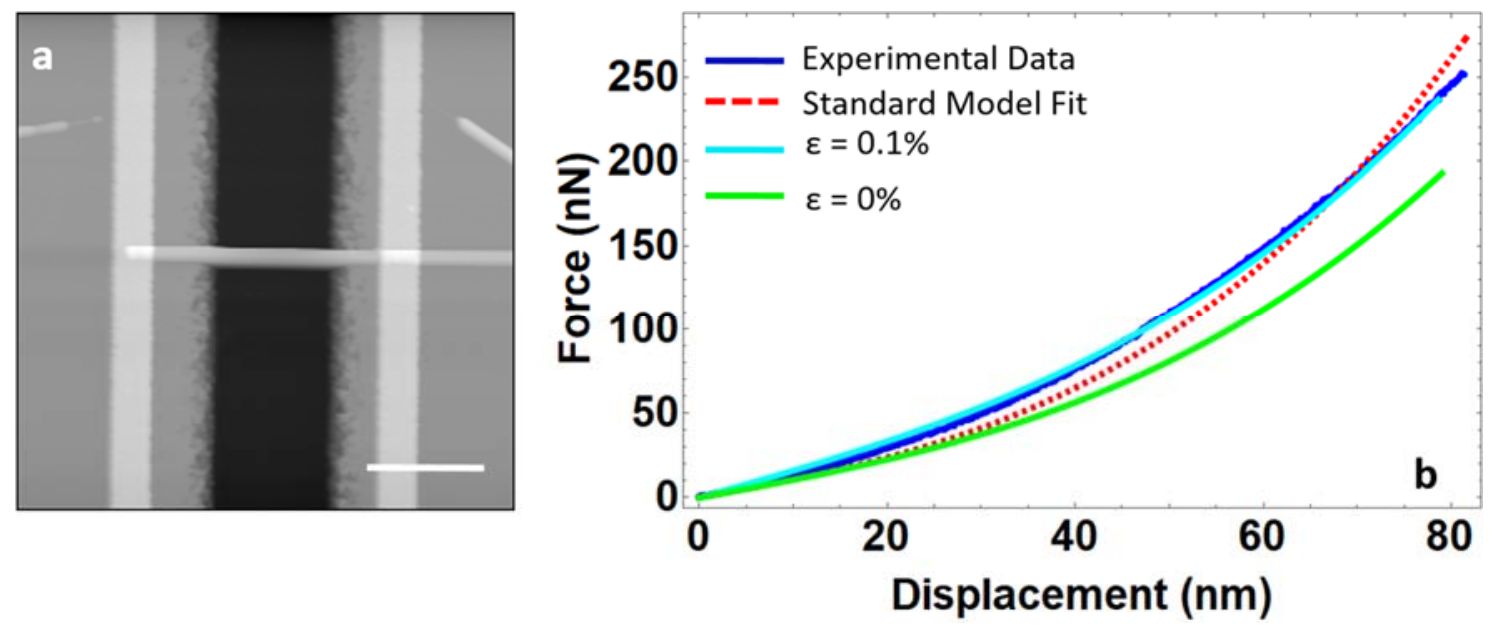

Figure 5. Effect of initial strain on F-d curve. a, Solvent-deposited AgNW deposited over a trench with electron beam lithography defined metallised clamps, scale bar $2 \mu \mathrm{m}$. b, Blue curve shows experimental $F-d$ response of AgNW in a, dashed red curve shows the fit of the standard model to the blue curve, cyan blue curve shows a synthetic $F-d$ curve for the NW in a if affected by an initial strain of $0.1 \%$ and the green curve shows the expected $F-d$ response for the same NW not affected by an initial strain.

Raman measurements on strain engineered SiNWs. Raman is a well-established method to measure the stress state of nanoscale materials but cannot be used to study metal NWs. We show that by coating SiNWs (shown earlier to be unaffected by solvent processing) with a nanoscale polymer film it is possible to engineer the final stress state of the material. SiNWs coated with 3-4 nm PVP layer (see Supplementary Figure S1 c) have similar adhesion properties to AgNWs when solvent deposited (dropcast or ink-jet). PVP-coated SiNWs were dry deposited onto a quartz substrate and the Raman frequency shift associated with individual NWs recorded before and after solvent exposure (see methods section). Figure 6a shows one such wire, which is about $8.5 \mu \mathrm{m}$ long. We find that after exposure to IPA there is a clear redshift of the Raman Si line near $520 \mathrm{~cm}^{-1}$, which is indicative of tensile stress ${ }^{23}$. From the measured red-shift of $0.5 \mathrm{~cm}^{-1}$ between Figure $6 \mathrm{~b}$ and $\mathrm{c}$, we estimate a strain level of about $0.1 \%$ when compared with studies on uniaxially strained silicon ${ }^{23}$. These studies also demonstrated that the strain level scales linearly with the Raman shift ${ }^{22}$. Measurements from other PVP-coated SiNWs are shown in Table S1. In each case the solvent treated wires are under tensile stress and we find that the strain is larger for the shorter wires (see inset Figure 
6a), reaching values of $0.6 \%$ for the shortest wires studied here ${ }^{22}$. The origin of this length dependence is not understood at present. We speculate that in the case of longer wires, adhesion does not occur uniformly along the length of the wires and that different parts of the wire adhere at different times preventing the full accumulation of stress along the length of the wire, which seems to be the case for smaller wires. However, these results clearly show that it is possible to engineer the stress state of single NWs by controlling the surface coating and solvent exposure.
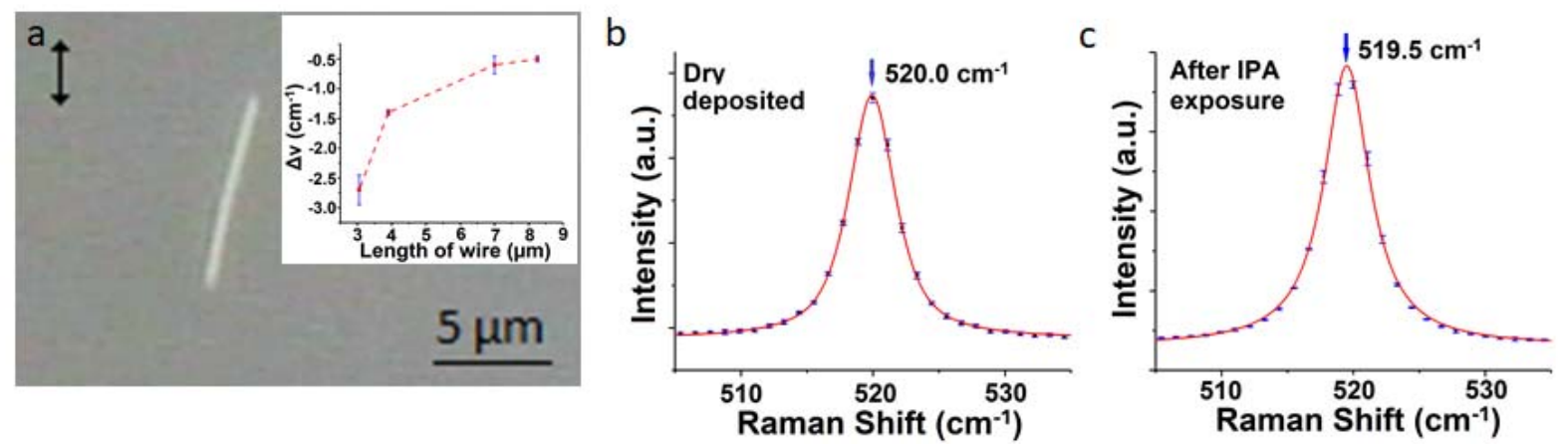

Figure 6. Raman Scattering Measurements on SiNWs. a Optical microscope image showing a $8.5 \mu \mathrm{m} \mathrm{SiNW}$ under study. Arrow indicates the polarization direction of excitation light. $\mathbf{b}$ Raman spectrum of the dry deposited Si NW. c Raman spectrum of SiNW after IPA treatment showing a clear red-shift indicative of tensile stress. Raman spectra are an average of three spectra recorded along the wire length. Inset Raman line shift versus wire length showing that shorter wires exhibit greater strain.

\section{CONCLUSIONS}

We have shown that surface stabilisation layers on nanowires swell in certain solvent media. These swollen layers cause an elongation of the nanowire, resulting in measured strains up to $0.6 \%$, with larger values for shorter wires. The stabilisation layer is also responsible for enhanced wire-substrate adhesion, due to an increased contact area between the solventswollen surface layer and the substrate. Strong adhesion prevents relaxation of solvent induced stress states in nanomaterials. This is particularly important since material properties such as 
the band gap, refractive index and carrier mobility are strongly modulated by stress. This work shows that solvent processed nanomaterials are always prone to the incorporation of stress. Particular care should be exercised in the use of solvents to prepare nanomaterials for characterisations, for example solvent drop-casting of materials for TEM analysis, and in the choice of solvent for ink-jet printing applications. Conversely, the judicious choice of surface passivation layer and solvent provides a means to engineer the stress state and hence the properties of nanoscale materials.

\section{ASSOCIATED CONTENT}

\section{Supporting Information}

Four additional figures and one table are available as Supporting Information. This information is available free of charge at http://pubs.acs.org/.

\section{AUTHOR INFORMATION}

\section{Author Contributions}

S.M. wrote the manuscript, designed and carried out the experiments. C.R. prepared and characterised wires for Raman measurements and analysis was carried out by J.J.W. and J.F.D. E.K.M. conducted the TEM analysis. D.J.H synthesised the SiNWs used in this work. J.F.C. led the research in UNC Chapel Hill. J.J.B. co-wrote the manuscript and led the overall effort. S.M., J.E.S. and J.F.D discussed the results and contributed to the writing of the manuscript.

\section{Corresponding Author}

*Email: jboland@tcd.ie

\section{Notes}

The authors declare no competing financial interest. 


\section{ACKNOWLEDGEMENTS}

This work was financially supported in part by a research grant from Science Foundation Ireland (12/IA/1482), the European Research Council under the Advanced Grant 321160 and through the generous support of the Naughton Foundation. We acknowledge support from the Australian Research Council Centre of Excellence in Exciton Science (CE170100026) and the Australian Council grants scheme. We would also like to thank the staff at the Advanced Microscopy Lab (AML) at Trinity College Dublin.

\section{REFERENCES}

(1) Stevenson, A. P.; Blanco Bea, D.; Civit, S.; Antoranz Contera, S.; Iglesias Cerveto, A.; Trigueros, S. Three Strategies to Stabilise Nearly Monodispersed Silver Nanoparticles in Aqueous Solution. Nanoscale Res. Lett. 2012, 7 (1), 151.

(2) Aiken, J. D.; Finke, R. G. A Review of Modern Transition-Metal Nanoclusters: Their Synthesis, Characterization, and Applications in Catalysis. J. Mol. Catal. 1999, 145 (1-2), 1-44.

(3) Hang, J.; Shi, L.; Feng, X.; Xiao, L. Electrostatic and Electrosteric Stabilization of Aqueous Suspensions of Barite Nanoparticles. Powder Technol. 2009, 192 (2), 166170.

(4) Sun, Y.; Melillo, J. M.; Bowles, F. P.; Melillo, J. M.; Steudler, P. A.; Rayment, M.; Trumbore, S. E.; Amundson, R.; Bergh, J.; Linder, S.; et al. Shape-Controlled Synthesis of Gold and Silver Nanoparticles. Science. 2002, 298 (5601), 2176-2179.

(5) Demming, A. Excelling under Strain : Band Engineering in Nanomaterials. Nanotechnology 2013, 24 (33), 1-2.

(6) Qi, F.; Jin, G. Strain Sensing and Far-Infrared Absorption in Strained Graphene Quantum Dots. J. Appl. Phys. 2013, 114 (073509), 1-9.

(7) Yang, A.; Hryn, A. J.; Bourgeois, M. R.; Lee, W.; Hu, J.; Schatz, G. C.; Odom, T. W. Programmable and Reversible Plasmon Mode Engineering. Proc. Natl. Acad. Sci. U. S. A. 2016, 113 (50), 1-6.

(8) Yeo, P. S. E.; Loh, K. P.; Gan, C. K. Strain Dependence of the Heat Transport Properties of Graphene Nanoribbons. Nanotechnology 2012, 23 (49), 1-10.

(9) Jung, K.; Cho, M.; Zhou, M. Strain Dependence of Thermal Conductivity of [0001] Oriented GaN Nanowires. Appl. Phys. Lett. 2011, 98 (4), 1-3.

(10) Heidelberg, A.; Ngo, L. T.; Wu, B.; Phillips, M. A.; Sharma, S.; Kamins, T. I.; Sader, J. E.; Boland, J. J. A Generalized Description of the Elastic Properties of Nanowires. Nano Lett. 2006, 6 (6), 1101-1106.

(11) Wu, B.; Heidelberg, A.; Boland, J. J. Microstructure-Hardened Silver Nanowires. Nano Lett. 2006, 6 (3), 468-472. 
(12) Wen, B.; Sader, J. E.; Boland, J. J. Mechanical Properties of ZnO Nanowires. Phys. Rev. Lett. 2008, 101 (17), 1-4.

(13) Mills, S.; Sader, J. E.; Boland, J. J. Material Characterisation of Nanowires with Intrinsic Stress. Nanotechnology 2017, 28 (35), 1-8.

(14) Coskun, S.; Aksoy, B.; Unalan, H. E. Polyol Synthesis of Silver Nanowires : An Extensive Parametric Study. Cryst. Growth Des. 2011, 2 (06800), 2-5.

(15) Kim, F.; Sohn, K.; Wu, J. S.; Huang, J. X. Chemical Synthesis of Gold Nanowires in Acidic Solutions. J. Am. Chem. Soc. 2008, 130 (44), 14442-14443.

(16) Alkilany, A. M.; Nagaria, P. K.; Wyatt, M. D.; Murphy, C. J. Cation Exchange on the Surface of Gold Nanorods with a Polymerizable Surfactant: Polymerization, Stability, and Evaluation. Langmuir 2010, 26 (12), 9328-9333.

(17) Nandi, S.; Winter, H. H. Swelling Behavior of Partially Cross-Linked Polymers: A Ternary System. Macromolecules 2005, 38 (10), 4447-4455.

(18) Dušek, K.; Choukourov, A.; Dušková-Smrčková, M.; Biederman, H. Constrained Swelling of Polymer Networks: Characterization of Vapor-Deposited Cross-Linked Polymer Thin Films. Macromolecules 2014, 47 (13), 4417-4427.

(19) Wiley, B.; Sun, Y.; Xia, Y. Synthesis of Silver Nanostructures with Controlled Shapes and Properties. Acc. Chem. Res. 2007, 40 (10), 1067-1076.

(20) Chen, W.; Wang, C.; Yan, L.; Huang, L.; Zhu, X.; Chen, B.; Sant, H. J.; Niu, X.; Zhu, G.; Yu, K. N.; et al. Improved Polyvinylpyrrolidone Microneedle Arrays with NonStoichiometric Cyclodextrin. J. Mater. Chem. B 2014, 2 (12), 1699-1705.

(21) Roy, N.; Saha, N.; Kitano, T.; Saha, P. Novel Hydrogels of PVP-CMC and Their Swelling Effect on Viscoelastic Properties. J. Appl. Polym. Sci. 2010, 117, 1703-1710.

(22) Yaish, Y. E.; Calahorra, Y.; Shtempluck, O.; Kotchetkov, V. Three-Point Bending Analysis of Doubly Clamped Silicon Nanowire Beams; Young's Modulus, Initial Stress, and Crystal Orientation. J. Appl. Phys. 2015, 117 (16), 1-11.

(23) Peng, C. Y.; Huang, C. F.; Fu, Y. C.; Yang, Y. H.; Lai, C. Y.; Chang, S. T.; Liu, C. W. Comprehensive Study of the Raman Shifts in Strained Silicon and Germanium. J. Appl. Phys. 2009, 105 (8), 083537.

(24) Pinion, C. W.; Nenon, D. P.; Christesen, J. D.; Cahoon, J. F. Identifying Crystallization and Incorporation Limited Regimes During Vapor-Liquid-Solid Growth of Si Nanowires. ACS Nano 2014, 8 (6), 6081-6088.

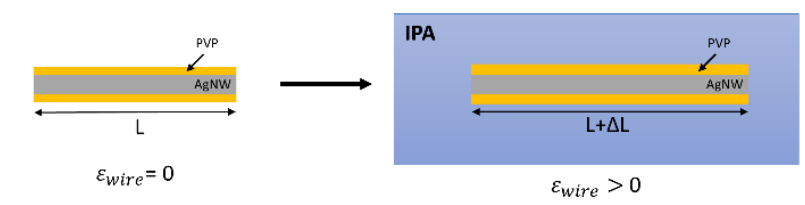

Dry Deposited:
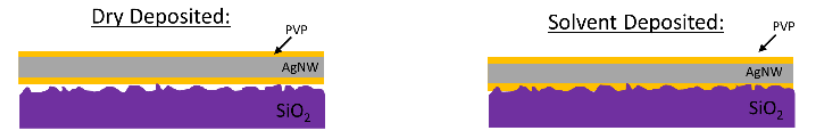


\section{Supporting Information}

\section{Solvent-Engineered Stress in Nanoscale Materials}

Shaun Mills ${ }^{\dagger,}$, , Chiara Rotella ${ }^{\dagger, \perp}$, Eoin K. McCarthy ${ }^{\perp}$, David J. Hill ${ }^{\phi}$, Jing-Jing Wang ${ }^{\perp}$, John F. Donegan ${ }^{\S, \perp}$, James F. Cahoon ${ }^{\phi}$, John E. Sader ${ }^{\ddagger}$, , \& John J. Boland ${ }^{*},+, \perp$

${ }_{\dagger}^{\dagger}$ School of Chemistry, Trinity College Dublin, Dublin 2, Ireland

${ }^{\S}$ School of Physics, Trinity College Dublin, Dublin 2, Ireland

${ }^{\perp}$ AMBER Research Centre and the Centre for Research on Adaptive Nanostructures and Nanodevices (CRANN), Trinity College Dublin, Dublin 2, Ireland

$\phi$ Department of Chemistry, University of North Carolina at Chapel Hill, Chapel Hill, North Carolina 27599-3290, United States

${ }^{\nabla}$ ARC Centre of Excellence in Exciton Science, School of Mathematics and Statistics, University of Melbourne, Victoria, 3010, Australia

*E-mail: jboland@tcd.ie 


\section{TEM images of AgNW and SiNW}

TEM images showing (a) a AgNW surrounded by the PVP layer, (b) the presence of an oxide layer on a SiNW (b) and (c) a SiNW after the wire was coated with PVP showing the presence of both oxide and polymer layers
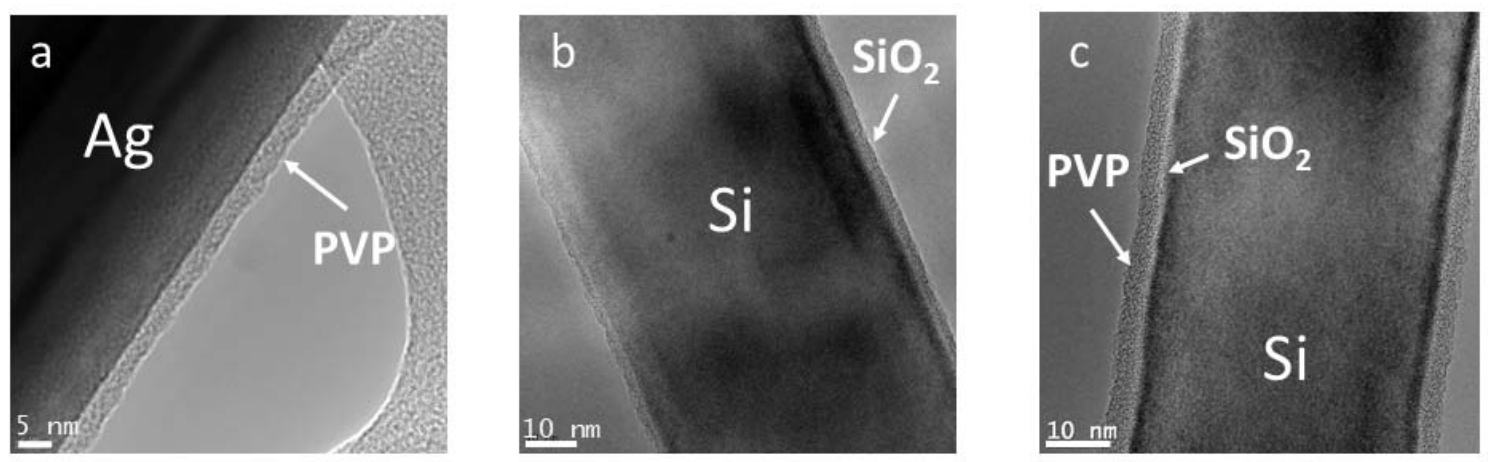

Figure S1. Nanowire TEM images. a, AgNW with a PVP coating b, SiNW with a native oxide outer shell and $\mathbf{c}$, SiNWs with a native oxide layer and a PVP coating. 


\section{Stress state comparison in AgNWs when processed using polar and non-polar solvents}

AgNWs are dispersed in a range of different solvents. Solvents are chosen depending on their ability to swell PVP. PVP is known to swell in polar solvents such as water and ethanol whereas it is non-swelling in non-polar solvents such as hexane and heptane. The aim of this measurement is to determine if the stress present in AgNWs varies depending on whether the solvent used to disperse them is capable of swelling the PVP surface layer. These NWs are dropcast over pre-defined trenches, mechanically clamped using Pt-EBID and measured using the 3-point bending technique. The $F-d$ curves are then fit using the standard model. In Fig. $\mathrm{S} 2$, $\mathrm{a}$ and $\mathrm{b}$ it can be observed that when AgNWs are dispersed in polar solvents such as water and ethanol they are subjected to stress due to the swelling of the PVP coating. In contrast, when they are dispersed in a non-polar solvent such as hexane or heptane they are not affected by stress and their $F-d$ response is accurately described by the standard model as shown in Fig. $\mathrm{S} 2 \mathrm{c}$ and $\mathrm{d}$.
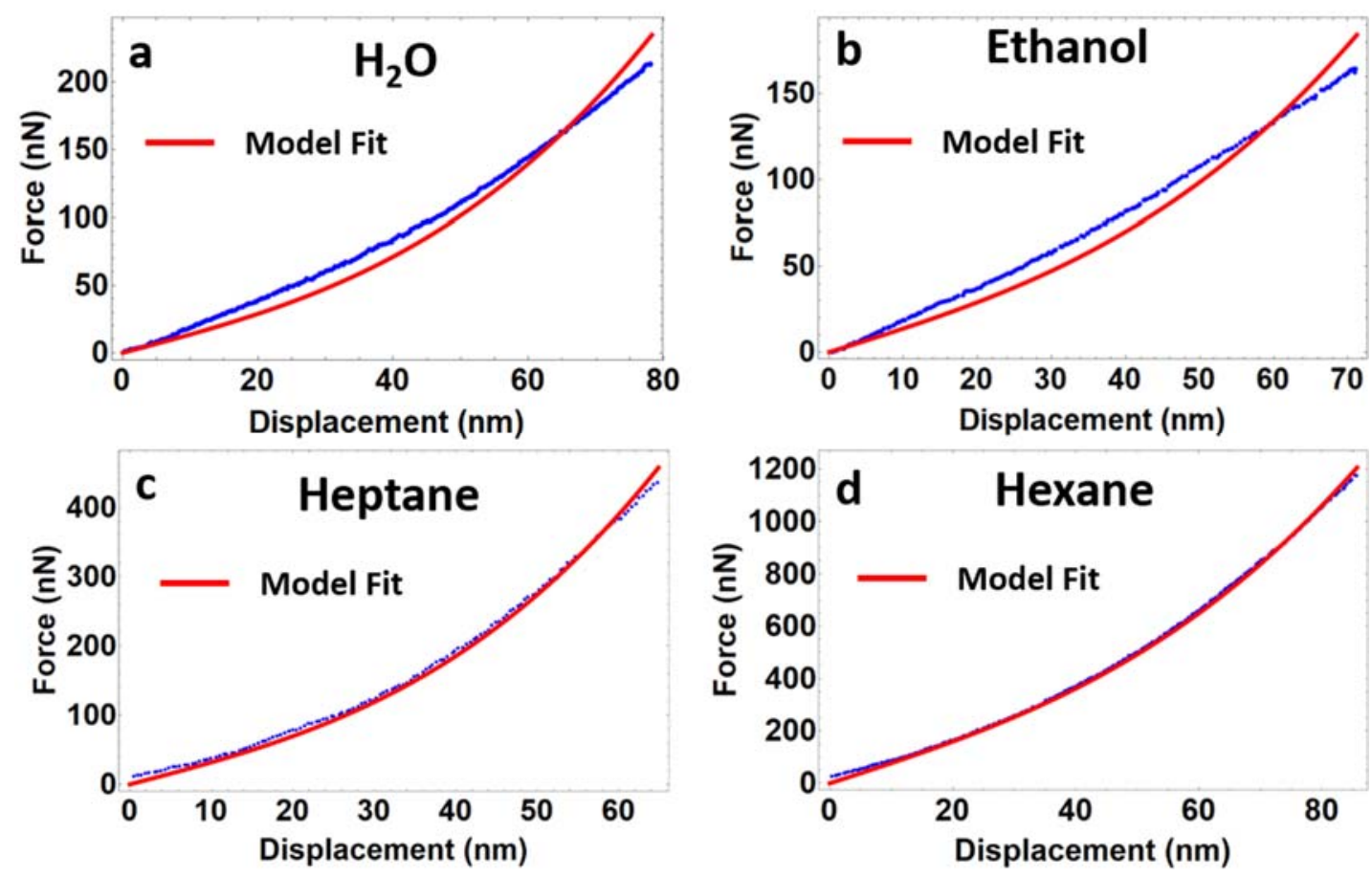

Figure S2. $F$ - $d$ curves of AgNWs processed using different solvents. F- $d$ curves for AgNWs when deposited using different solvents and fit to the standard model. The solvents used are; a, Water, b, Ethanol, c, Heptane, d, Hexane. 


\section{Variation in adhesion of AgNWs when processed using polar and non-polar solvents}

The adhesion of AgNWs dispersed in polar and non-polar solvents is investigated by dispersing AgNWs in various solvents, dropcasting them on a blank $\mathrm{SiO}_{2}$ substrate and determining their adhesion by manipulation in air with an AFM tip. From Fig. S3 b and $d$ it is shown that when AgNWs are deposited in polar solvents such as water and ethanol, that the NWs are strongly adhered to the surface. NW fracture is generally observed. When deposited in non-polar solvents such as hexane and heptane, AgNWs adhesion to $\mathrm{SiO}_{2}$ is reduced as the NWs are mobile on the surface as seen in $\mathrm{f}$ and $\mathrm{g}$. The enhanced adhesion of the NWs when dispersed in polar solvents is due to the proposed softening of the polymer upon swelling which allows it to better conform to surface due to an increased contact area. In contrast, manipulations performed directly in the solvent caused the wires to become dislodged from the substrate due to poor adhesion.

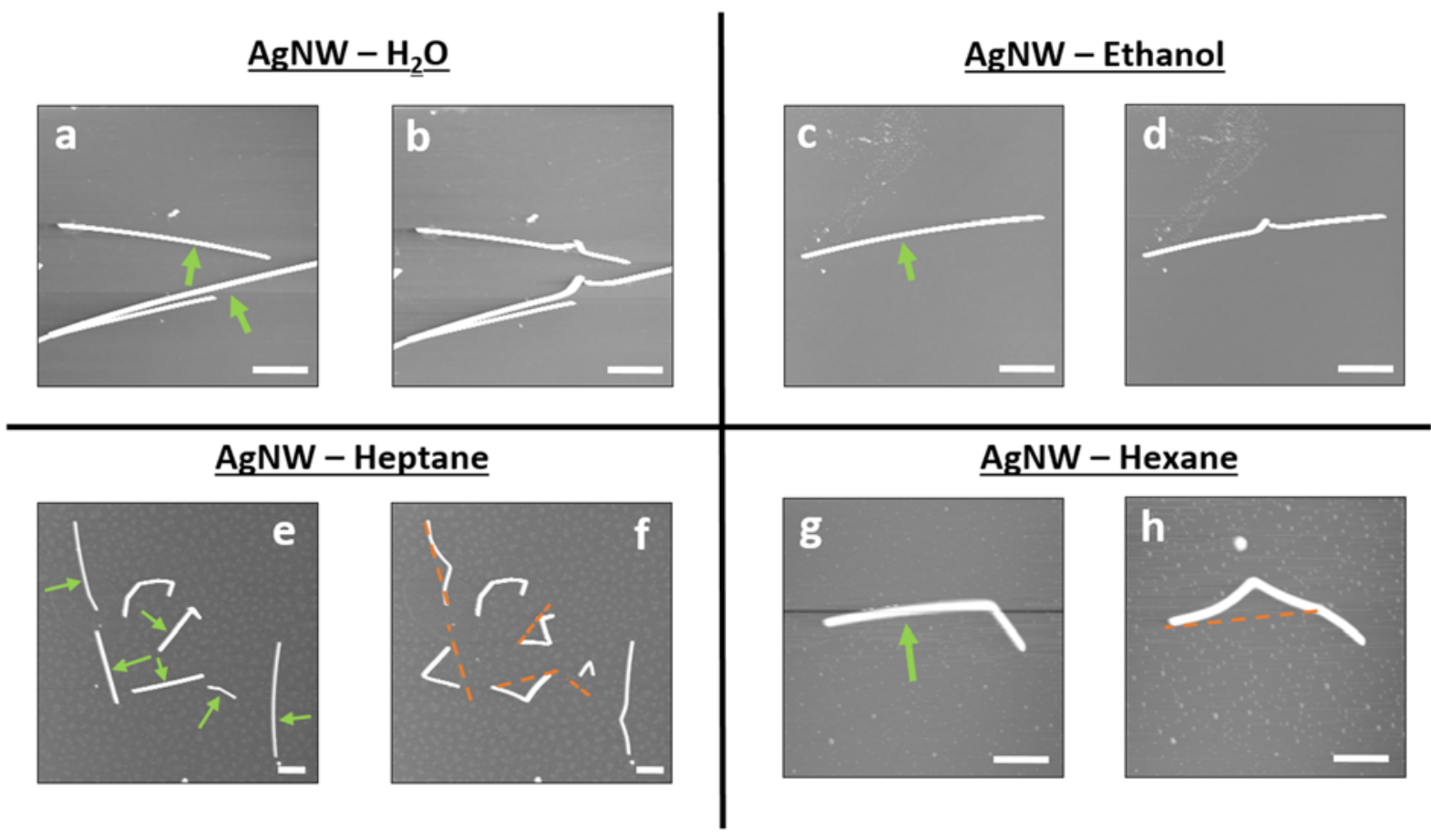

Figure S3. Adhesion of $\mathrm{AgNW}$ to $\mathrm{SiO}_{2}$ when processed using different solvents. AFM images of $\mathrm{AgNW}$ dispersed on $\mathrm{SiO}_{2}$ before and after being manipulated with an AFM tip. NWs were deposited using both polar and non-polar solvents, a, Water, c, Ethanol, e, Heptane, g, Hexane. Images a,c,e,g, are wires before manipulation with the green arrows defining the loading path. The corresponding images after manipulation are shown in $\mathbf{b}, \mathbf{d}, \mathbf{f}$, $\mathbf{h}$, all scale bars $2 \mu \mathrm{m}$. 


\section{Enhanced adhesion and stress in AuNWs due to solvent deposition}

AuNWs are deposited on $\mathrm{SiO}_{2}$ substrate using both solvent and dry-deposition methods. They are synthesised by Sigma-Aldrich in $\mathrm{H}_{2} \mathrm{O}$ and so are dropcast in $\mathrm{H}_{2} \mathrm{O}$. As can be observed in Fig. S4 b, when AuNWs are deposited in $\mathrm{H}_{2} \mathrm{O}$ and manipulated they are strongly adhered to the $\mathrm{SiO}_{2}$ substrate which results in wire fracture. When dry-deposited they are weakly adhered to the substrate as shown in Fig. S4 d. Furthermore, when they are solvent-deposited over a trench, mechanically clamped with Pt-EBID and manipulated they are affected by stress. This can be recognised by the poor fit of the experimental data to the standard model as shown in Fig. S4 e. Evidence of the CTAB surface coating on these AuNWs is shown in the TEM image in Fig. S4 $\mathrm{f}$.

\section{AuNW - Solvent Deposited}
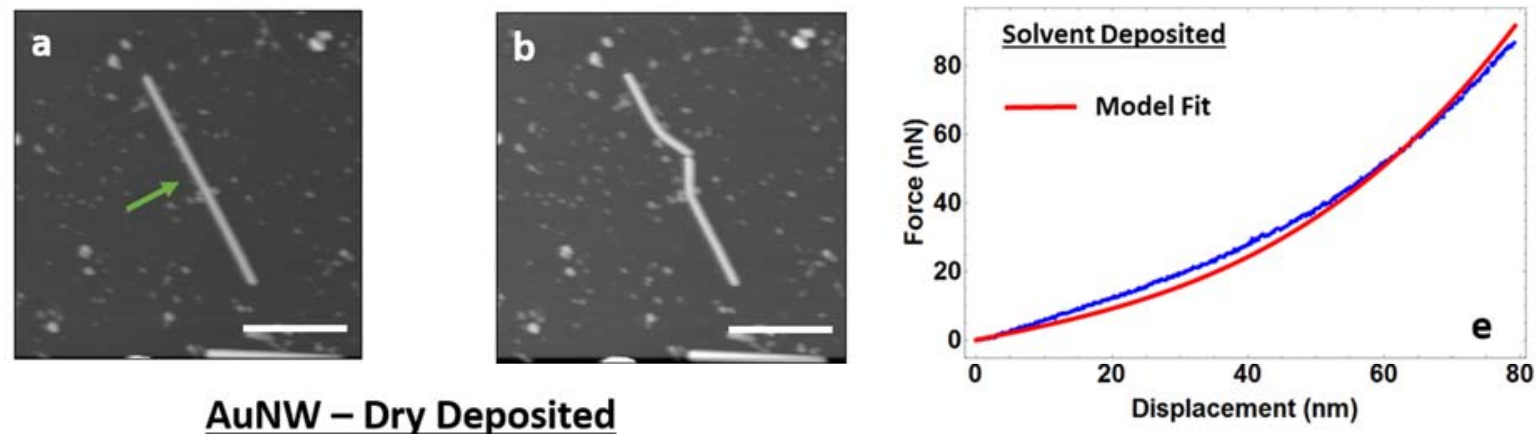

\section{AuNW - Dry Deposited}
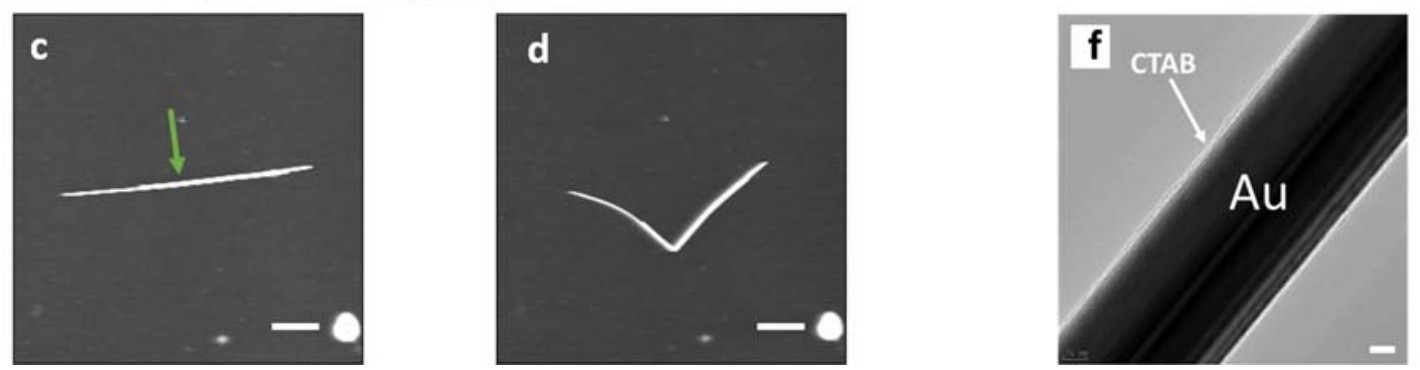

Figure S4. AuNW experiment. AFM images of AuNWs deposited on $\mathrm{SiO}_{2}$ a, solventdeposited, c, dry-deposited, with the resulting corresponding AFM images in b, d after AFM manipulation, all scale bars $1 \mu \mathrm{m}$. e, F- $d$ response of solvent-deposited AuNW in doublyclamped beam configuration fit to the standard (zero-stress) model. f, TEM image showing CTAB coating on the surface of the AuNW, scale bar $10 \mathrm{~nm}$. 


\section{Raman Scattering Measurements on SiNWs}

Raman measurements of SiNWs with PVP coating layer deposited on quartz substrate using dry-deposition methods and after the same wire is exposed to isopropanol (IPA) solvent. We use a wavelength of $488 \mathrm{~nm}$, the spectrometer is a Renishaw Invia spectrometer with a $3000 / \mathrm{mm}$ grating, which has an instrumental resolution of better than $0.2 \mathrm{~cm}^{-1}$. The probe size is about $0.5 \mu \mathrm{m}$ diameter and three measurements were performed on each wire.

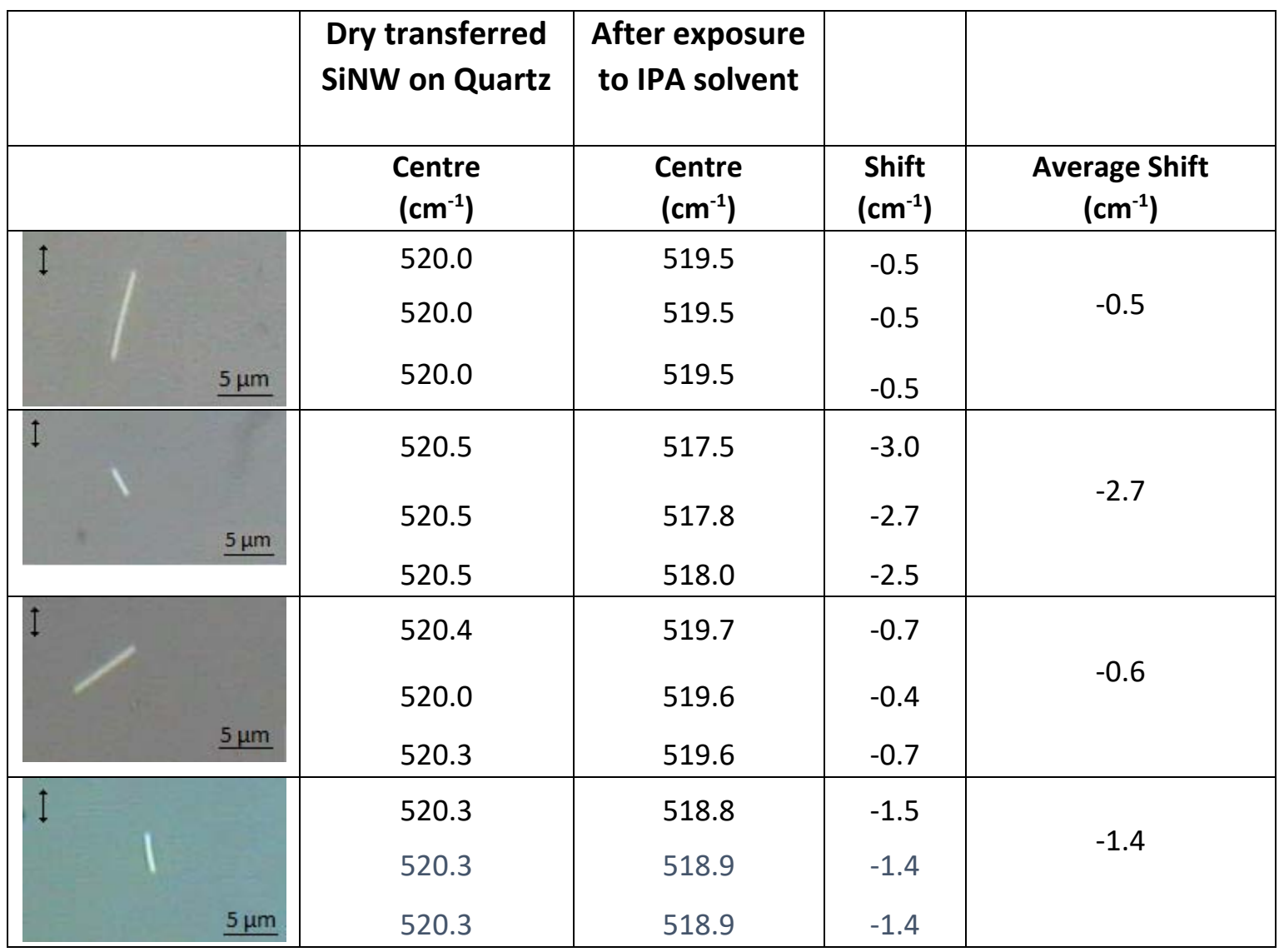

Table S1. Raman Scattering Measurements on SiNWs. Results show results for four different wires of different lengths before and after solvent exposure. For each wire three measurements are recorded at different position along the wire length. The results are summarised in the inset of Fig. 6 and show that shorter wires exhibit a greater Raman shift indicating a greater tensile strain in these cases. 